\title{
Perspective
}

\section{Translational Studies on Glutamate and Dopamine Neurocircuitry in Addictions: Implications for Addiction}

\section{Treatment}

\author{
Evelyn K Lambe*,' and Tony P George ${ }^{2,3}$ \\ 'Department of Physiology and Department of Obstetrics and Gynecology, University of Toronto, Toronto, ON, Canada; ${ }^{2}$ Schizophrenia and \\ Addictions Programs, Centre for Addiction and Mental Health (CAMH), Toronto, ON, Canada; ${ }^{3}$ Departments of Psychiatry and Psychology, \\ University of Toronto, Toronto, ON, Canada
}

\begin{abstract}
New research suggests that the modulation of dopamine neurocircuitry by glutamate plays a key role in the development of nicotine and other addictions. For example, manipulation of glutamatergic pathways can alter the mood-enhancing and reinforcing properties of nicotine. These glutamatergic pathways are also sensitive to manipulation by other drugs of abuse. The studies described in this special issue of Neuropsychopharmacology bring together rodent studies with translational work in humans to enhance our understanding of the cellular mechanisms underlying the subjective and objective effects of drugs of abuse. These studies suggest new therapeutic targets based on central glutamate systems that may lead to the development of novel and more effective treatments for addictive disorders. Neuropsychopharmacology (2009) 34, 255-256; doi:I0.1038/npp.2008.196; published online 5 November 2008
\end{abstract}

Keywords: glutamate; dopamine; ventral tegmental area; nucleus accumbens; prefrontal cortex; nicotine

The series of articles published in this issue of Neuropsychopharmacology show that glutamate modulates dopamine neurons in the ventral tegmental area (VTA) in ways that are important for understanding addiction to nicotine and other drugs of abuse. The mood enhancing and reinforcing properties of nicotine can be altered by a number of factors that change glutamatergic signaling in the VTA. Research into the cellular mechanisms through which glutamate excites dopamine neurocircuits is critical to identify new avenues for translational work in the treatment of addictions.

Kenny et al (2008) demonstrate the relevance of glutamatergic NMDA receptors in the VTA for the motivation to self-administer nicotine. Local infusion of an NMDA receptor antagonist into the VTA decreased selfadministration of nicotine, and systemic application of this glutamatergic drug reversed the hedonic valence of nicotine from positive to negative. The importance of NMDA receptors is further probed in a human translational study by Jackson et al (2008) for their role in mediating the 'buzz' elicited by cigarette smoking. This research suggests that glutamatergic and nicotinic mechanisms may play distinct roles in the subjective and cognitive effects of smoking.

*Correspondence: Dr EK Lambe, Department of Physiology, University of Toronto, I King's College Circle, Toronto, ON M5S IA8, Canada, Tel: + 4169460910 , Fax: + 416978 4940,

E-mail: evelyn.lambe@utoronto.ca

Received 29 September 2008; accepted 3 October 2008
Taken together, these findings have important consequences for designing pharmacotherapies to reduce the reinforcing and rewarding actions of nicotine in the clinical management of tobacco addiction. This research suggests that a focus on glutamatergic pathways may allow the rewarding properties of nicotine to be dissociated from its cognitive actions.

The manuscripts by Xiao et al (2008) and Heikkinen et al (2008) explore the ability of two other addictive substances - ethanol and benzodiazepines - to alter glutamatergic signaling in the VTA. These findings underscore the potential for rapid and long-lasting modulation of dopaminergic neurons by commonly used substances such as alcohol and sedative hypnotics, as illustrated by the schematics in Figure 1.

Although there has been controversy about the role of nonnicotine components of tobacco smoke (eg tar) and psychological factors in the reinforcing properties of cigarette smoking, Brody et al (2008) demonstrate that nicotine itself is critical for the mood-altering effects of cigarettes. These human PET imaging experiments used regular and denicotinized cigarettes to illustrate that the nicotine in cigarettes is necessary for both dopamine release in ventral striatum and mood improvement from smoking (Figure 1). These results add to the growing body of literature that support the potential role of nicotinic receptor modulation in the treatment of mood disorders and comorbid nicotine addiction (George et al, 2008; Mineur et al, 2007). 


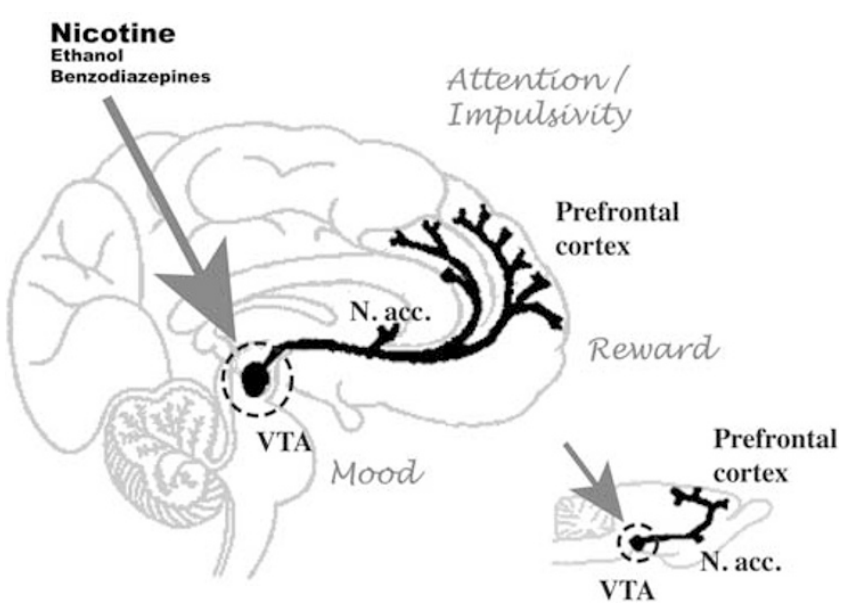

Figure I Schematics of human and rodent brains depict how the dopamine projections of the ventral tegmental area (VTA) innervate the nucleus accumbens ( $\mathrm{N}$. acc.) and the prefrontal cortex. These dopamine projections have been shown to be important for the modulation of mood, reward, and attention. The series of articles in this issue of the journal highlight the importance of the glutamatergic modulation of dopamine neurons in the VTA in understanding nicotine and other drug addictions. (Adapted from Schultz, 1999; Bailey, 2005.)

The final article in this issue demonstrates that the neurocircuitry of the VTA is particularly vulnerable to disruption during adolescence, and this disruption can lead to long-lasting cognitive deficits. Counotte et al (2008) show that adolescent nicotine exposure can result in seemingly permanent changes to dopamine neuron excitability in rats. In adulthood, these rodents exhibit diminished attentional performance and an increase in impulsive behaviors (Figure 1).

Taken together, the contributions from these investigations point to the need to focus more intensely on the glutamatergic modulation of VTA neurocircuitry in addictive disorders. The vulnerability of these pathways during development and the potential for interactions with other drugs suggests new ways to understand the mechanisms underlying addiction. The studies in this issue lay a foundation for understanding the glutamatergic and dopaminergic microcircuitry of the VTA, and they show how the VTA is sensitive to drug exposure throughout neural development. This information is essential to identify novel treatments to restore the normal modulation of the VTA dopamine neurons in people with addictions. The development of glutamatergic agents to treat addiction is still in its infancy; however, there is already intriguing evidence from preliminary clinical studies in subjects addicted to cocaine (LaRowe et al, 2007), tobacco (Anthenelli et al, 2008), and alcohol (Johnson et al, 2007) showing that such modulation of central glutamatergic function may be appropriate for both the initiation of abstinence and the prevention of relapse. Patients with addictions and their families will welcome advances in these areas because new and more effective treatments are essential to alleviate the economic and personal toll wrought by drug addiction in modern society.

\section{DISCLOSURES}

Dr Lambe is supported by a Canada Research Chair in Developmental Cortical Neurophysiology, the Canada Foundation for Innovation (CFI) and the Canadian Institutes of Health Research (CIHR). Dr George is a consultant on addiction medications for Pfizer, Eli Lily, Prempharm, and Evotec, and has grant support from Pfizer, Sepracor, Targacept Inc., the National Institute on Drug Abuse (NIDA; grants K02-DA-16611 and R01-DA-15757), the National Alliance for Research on Schizophrenia and Depression (NARSAD), CIHR, CFI and the Endowed Chair in Addiction Psychiatry at the University of Toronto.

\section{REFERENCES}

Anthenelli RM, Blom TJ, McElroy SL, Keck Jr PE (2008). Preliminary evidence for gender-specific effects of topiramate as a potential aid to smoking cessation. Addiction 103: 687-694.

Bailey C (2005). E-Journal of the British Pharmacological Society. pA2 Online 3: 3.

Brody AL, Mandelkern MA, Olmstead RE, Allen-Martinez Z, Scheibal D, Abrams AL et al (2008). Ventral striatal dopamine release in response to smoking a regular vs a denicotinized cigarette. Neuropsychopharmacol 34: 282-289.

Counotte DS, Spijker S, Van de Burgwal LH, Hogenboom F, Schoffelmeer ANM, De Vries TJ et al (2008). Long-lasting cognitive deficits resulting from adolescent nicotine exposure in rats. Neuropsychopharmacol 34: 299-306.

George TP, Vessicchio JC, Sacco KA, Weinberger AH, Shytle RD (2008). Nicotinic antagonist augmentation of selective serotonin reuptake inhibitor-refractory major depressive disorder: a preliminary study. J Clin Psychopharmacol 28: 340-344.

Heikkinen AE, Moykkyen TP, Korpi ER (2008). Long-lasting modulation of glutamatergic transmission in VTA dopamine neurons after a single dose of benzodiazepine agonists. Neuropsychopharmacol 34: 290-298.

Jackson A, Nesic J, Groombridge C, Clowry O, Rusted J, Duka T. (2008). Differential involvement of glutamatergic mechanisms in the cognitive and subjective effects of smoking. Neuropsychopharmacol 34: 257-265.

Johnson BA, Rosenthal N, Capece JA, Wiegand F, Mao L, Beyers K et al (2007). Topiramate for treating alcohol dependence: a randomized controlled trial. JAMA 298: 1641-1651.

Kenny PJ, Chartoff E, Roberto M, Carlezon Jr WA, Markou A (2008). NMDA receptors regulate nicotine-enhanced brain reward function and intravenous nicotine self-administration: role of the ventral tegmental area and central nucleus of the amygdala. Neuropsychopharmacol 34: 266-281.

LaRowe SD, Myrick H, Hedden S, Mardikian P, Saladin M, McRae A et al (2007). Is cocaine desire reduced by N-acetylcysteine? Am J Psychiatry 164: 1115-1117.

Mineur YS, Somenzi O, Picciotto MR (2007). Cytisine, a partial agonist of high-affinity nicotinic acetylcholine receptors, has antidepressant-like properties in male C57BL/6J mice. Neuropharmacology 52: 1256-1262.

Schultz W (1999). The reward signal of midbrain dopamine neurons. News Physiol Sci 14: 249-255.

Xiao C, Shao XM, Olive MO, Griffin III WC, Li K-Y, Krnjevic K et al (2008). Ethanol facilitates glutamatergic transmission to dopamine neurons in the ventral tegmental area. Neuropsychopharmacol 34: 307-318. 